\title{
MAGIC OBSERVATIONS OF THE UNIDENTIFIED $\gamma$-RAY SOURCE TeV J2032+4130
}

J. Albert,${ }^{1}$ E. Aliu,${ }^{2}$ H. Anderhub,${ }^{3}$ P. Antoranz,${ }^{4}$ C. Baixeras,${ }^{5}$ J. A. Barrio,${ }^{4}$ H. Bartko,${ }^{6}$ D. Bastieri, ${ }^{7}$ J. K. Becker,${ }^{8}$ W. Bednarek, ${ }^{9}$ K. Berger,${ }^{1}$ C. Bigongiari, ${ }^{7}$ A. Biland, ${ }^{3}$ R. K. Bock,${ }^{6,7}$ G. Bonnoli, ${ }^{10}$ P. Bordas, ${ }^{11}$ V. Bosch-Ramon, ${ }^{11}$ T. Bretz,${ }^{1}$ I. Britvitch,${ }^{3}$ M. Camara, ${ }^{4}$ E. Carmona,${ }^{6}$ A. Chilingarian, ${ }^{12}$ S. Commichau, ${ }^{3}$ J. L. Contreras, ${ }^{4}$ J. Cortina, ${ }^{2,13}$ M. T. Costado, ${ }^{14,15}$ V. Curtef, ${ }^{8}$ F. Dazzi, ${ }^{7}$ A. De Angelis,${ }^{16}$ C. Delgado, ${ }^{14}$ R. De los Reyes, ${ }^{4}$ E. Domingo-Santamaría, ${ }^{2}$ B. De Lotto, ${ }^{16}$ M. De Maria,${ }^{16}$ F. De Sabata,${ }^{16}$ D. Dorner, ${ }^{1}$ M. Doro, ${ }^{7}$ M. Errando, ${ }^{2}$ M. Fagiolini, ${ }^{10}$ D. Ferenc, ${ }^{17}$ E. Fernández,${ }^{2}$ R. Firpo, ${ }^{2}$ M. V. Fonseca,${ }^{4}$ L. Font,${ }^{5}$ N. Galante, ${ }^{6}$ R. J. García-López, ${ }^{14,15}$ M. Garczarczyk,${ }^{6}$ M. Gaug,${ }^{14}$ F. Goebel,${ }^{6}$ M. Hayashida, ${ }^{6}$ A. Herrero, ${ }^{14,15}$ D. Höhne, ${ }^{1}$ J. Hose, ${ }^{6}$ C. C. Hsu, ${ }^{6}$ S. Huber,${ }^{1}$ T. Jogler,${ }^{6}$ R. Kosyra,${ }^{6}$ D. Kranich,${ }^{3}$ A. Laille, ${ }^{17}$ E. Leonardo, ${ }^{10}$ E. Lindfors,${ }^{18}$ S. Lombardi, F. Longo, ${ }^{16}$ M. López, ${ }^{4}$ E. Lorenz,${ }^{3,6}$ P. Majumdar,${ }^{6}$ G. Maneva,${ }^{19}$ N. Mankuzhiyil,${ }^{16}$ K. Mannheim, ${ }^{1}$ M. Mariotti ${ }^{7}$ M. Martínez, ${ }^{2}$ D. Mazin,${ }^{2}$ C. Merck,${ }^{6}$ M. Meucci,${ }^{10}$ M. Meyer,${ }^{1}$ J. M. Miranda, ${ }^{4}$ R. Mirzoyan, ${ }^{6}$ S. Mizobuchi, ${ }^{6}$ A. Moralejo, ${ }^{2}$ D. Nieto,${ }^{4}$ K. Nilsson, ${ }^{18}$ J. Ninkovic, ${ }^{6}$ E. OÑa-Wilhelmi,${ }^{2,13,20}$ N. Otte,${ }^{6,21}$ I. Oya ${ }^{4}$ M. Panniello,${ }^{14,22}$ R. Paoletti,${ }^{10}$ J. M. Paredes, ${ }^{11}$ M. Pasanen,${ }^{18}$ D. Pascoli,${ }^{7}$ F. Pauss,${ }^{3}$ R. Pegna,${ }^{10}$ M. Persic,${ }^{16,23}$ L. Peruzzo, ${ }^{7}$ A. Piccioli,${ }^{10}$ E. Prandini, ${ }^{7}$ N. Puchades, ${ }^{2}$ A. Raymers,${ }^{12}$ W. Rhode, ${ }^{8}$ M. Ribó,${ }^{11}$ J. Rico, ${ }^{2}$ M. Rissi,${ }^{3}$ A. Robert,${ }^{5}$ S. RÜgamer, ${ }^{1}$ A. Saggion, ${ }^{7}$ T. Y. Saito, ${ }^{6}$ A. Sánchez, ${ }^{5}$ P. Sartori,${ }^{7}$ V. Scalzotto, ${ }^{7}$ V. Scapin, ${ }^{16}$ R. Schmitt, ${ }^{1}$ T. Schweizer, ${ }^{6}$ M. Shayduk,${ }^{6,21}$ K. Shinozaki,${ }^{6}$ S. N. Shore, ${ }^{24}$ N. Sidro, ${ }^{2}$ A. Sillanpää, ${ }^{18}$ D. Sobczynska, ${ }^{9}$ F. Spanier, ${ }^{1}$ A. Stamerra, ${ }^{10}$ L. S. Stark, ${ }^{3}$ L. Takalo,${ }^{18}$ P. Temnikov,${ }^{19}$ D. Tescaro, ${ }^{2}$ M. Teshima, ${ }^{6}$ D. F. Torres,,${ }^{13,25}$ N. Turini, ${ }^{10}$ H. Vankov, ${ }^{19}$ A. Venturini,${ }^{7}$ V. Vitale,${ }^{16}$ R. M. Wagner, ${ }^{6}$ W. Wittek, ${ }^{6}$ F. Zandanel,${ }^{7}$ R. Zanin, ${ }^{2}$ and J. Zapatero ${ }^{5}$ Received 2007 December 14; accepted 2008 January 15; published 2008 February 5

\section{ABSTRACT}

We observed the first known very high energy (VHE) $\gamma$-ray-emitting unidentified source, TeV J2032+4130, for $94 \mathrm{hr}$ with the MAGIC telescope. The source was detected with a significance of $5.6 \sigma$. The flux, position, and angular extension are compatible with the previous ones measured by the HEGRA telescope system 5 years ago. The integral flux amounts to $\left(4.5 \pm 0.3_{\text {stat }} \pm 0.35_{\text {sys }}\right) \times 10^{-13}$ photons $\mathrm{cm}^{-2} \mathrm{~s}^{-1}$ above $1 \mathrm{TeV}$. The source energy spectrum, obtained with the lowest energy threshold to date, is compatible with a single power law with a hard photon index of $\Gamma=-2.0 \pm 0.3_{\text {stat }} \pm 0.2_{\text {sys }}$.

Subject heading: gamma rays: observations

\section{INTRODUCTION}

The $\mathrm{TeV}$ source $\mathrm{J} 2032+4130$ (Aharonian et al. 2002) was the first unidentified very high energy (VHE) $\gamma$-ray source, and also the first discovered extended $\mathrm{TeV}$ source, likely to be Galactic.

Intensive observational campaigns at different wavelengths

${ }^{1}$ Universität Würzburg, D-97074 Würzburg, Germany.

${ }^{2}$ IFAE, Edifici Cn., E-08193 Bellaterra (Barcelona), Spain.

${ }^{3}$ ETH Zurich, CH-8093, Switzerland.

${ }^{4}$ Universidad Complutense, E-28040 Madrid, Spain.

${ }^{5}$ Universitat Autònoma de Barcelona, E-08193 Bellaterra, Spain.

${ }^{6}$ Max-Planck-Institut für Physik, D-80805 Munich, Germany.

${ }^{7}$ Università di Padova and INFN, I-35131 Padova, Italy.

${ }^{8}$ Universität Dortmund, D-44227 Dortmund, Germany.

${ }^{9}$ University of Łódź, PL-90236 Lodz, Poland.

${ }^{10}$ Università di Siena, and INFN Pisa, I-53100 Siena, Italy.

${ }^{11}$ Universitat de Barcelona, E-08028 Barcelona, Spain.

${ }^{12}$ Yerevan Physics Institute, AM-375036 Yerevan, Armenia.

${ }^{13}$ Corresponding author.

${ }^{14}$ Inst. de Astrofisica de Canarias, E-38200 La Laguna, Tenerife, Spain.

${ }^{15}$ Depto. de Astrofisica, Universidad, E-38206 La Laguna, Tenerife, Spain.

${ }^{16}$ Università di Udine, and INFN Trieste, I-33100 Udine, Italy.

${ }^{17}$ University of California, Davis, CA 95616.

${ }^{18}$ Tuorla Observatory, Turku University, FI-21500 Piikkiö, Finland.

${ }^{19}$ Inst. for Nucl. Research and Nucl. Energy, BG-1784 Sofia, Bulgaria.

${ }^{20}$ Current address: APC (CNRS) Paris, France.

${ }^{21}$ Humboldt-Universität zu Berlin, D-12489 Berlin, Germany.

${ }^{22}$ Deceased.

${ }^{23}$ INAF-Osservatorio Astronomico and INFN, I-34131 Trieste, Italy.

${ }^{24}$ Università di Pisa, and INFN Pisa, I-56126 Pisa, Italy.

${ }^{25}$ ICREA and Institut de Ciències de l'Espai (IEEC-CSIC), 08193 Barcelona, Spain. have been carried out on TeV J2032+4130. Butt et al. (2003) presented an analysis of the $\mathrm{CO}, \mathrm{H} \mathrm{I}$, and infrared emissions, together with first observations by Chandra (5 ks) and a reanalysis of VLA data. These observations showed that the TeV source region is positionally coincident with an outlying group of stars (from the Cygnus OB2 core), although they failed to identify a counterpart. Mukherjee et al. (2003) analyzed the same Chandra data and provided optical follow-up observations of several of the brightest X-ray sources, confirming that most were either $\mathrm{O}$ stars or foreground late-type stars. A deeper Chandra observation (50 ks; Butt et al. 2006) found hundreds of starlike sources and yet no diffuse X-ray counterpart emission.

A deep ( 50 ks) XMM-Newton exposure has also been obtained (Horns et al. 2007). After the subtraction of the contribution of known sources from the data, an extended X-ray emission region with a FWHM size of $\sim 12^{\prime}$ was reported. The centroid of the emission is colocated with the position of $\mathrm{TeV}$ $\mathrm{J} 2032+4130$ and was proposed as the counterpart of the $\mathrm{TeV}$ source. The question of whether the result reported by Horns et al. can be interpreted as a truly diffuse background, or whether it could be a result of unresolved X-ray sources, remains disputable.

Paredes et al. (2007) and Martí et al. (2007) have provided deep radio observations covering the $\mathrm{TeV} \mathrm{J} 2032+4130$ vicinity using the Giant Metrewave Radio Telescope and discovered a population of radio sources, some in coincidence with X-ray detections by Butt et al. (2006) and with optical/IR counterparts. At least three of these sources are nonthermal, and one 
TABLE 1

Observing Periods, Zenith Angle Ranges, AND OBSERVATION MOdes

\begin{tabular}{cccc}
\hline \hline Year & $\begin{array}{c}\text { Time } \\
(\mathrm{hr})\end{array}$ & $\begin{array}{c}\text { Zenith Angle } \\
(\mathrm{deg})\end{array}$ & Mode \\
\hline $2005 \ldots \ldots$ & 18.1 & $13-30$ & ON/OFF \\
$2006 \ldots \ldots$ & 60.1 & $11-44$ & Wobble \\
$2007 \ldots \ldots$ & 15.5 & $11-30$ & Wobble \\
\hline
\end{tabular}

has a hard X-ray energy spectrum. They found extended nonthermal diffuse emission in the radio band apparently connecting with one or two radio sources. It is yet to be determined whether one or more of these sources is similar to some of the known $\gamma$-ray binaries (e.g., Aharonian et al. 2006; Albert et al. 2006a).

Several theoretical explanations for the $\mathrm{TeV}$ emission from $\mathrm{J} 2032+4130$ have been given. Among them, those related to extragalactic counterparts, e.g., a radio galaxy (Butt et al. 2006) or a proton blazar (Mukherjee et al. 2003), face the difficulty of explaining the extended appearance of the source. Gammaray production in hypothetical jet termination lobes of Cyg X3 was explored (Aharonian et al. 2002), but the putative northern lobe of Cyg X-3 (now considered a mere thermal H II region; Martí et al. 2006) is far from the location of the $\mathrm{TeV}$ source. A yet unknown pulsar wind nebula (PWN) was proposed by Bednarek (2003), although no clear PWN signal was observed. A distant microquasar was proposed by Paredes et al. (2007), perhaps related to one of the X-ray/radio sources they discovered. If such an association is accepted, the extension of the source could be explained by the diffusion of accelerated particles into a hypothetical nearby molecular enhancement (see Bosch-Ramon et al. 2005). Torres et al. (2004) and Domingo-Santamaría \& Torres (2006) studied the relationship between the $\mathrm{TeV}$ emission and the known massive stars in the area, through the interaction of relativistic protons with wind ions. The distribution of stars in the neighborhood favors this interpretation (Butt et al. 2006). An explanation involving the excitation of giant dipole resonances of relativistic heavy nuclei in radiation-dominated environments has also been suggested (Anchordoqui et al. 2007).

\section{PREVIOUS VERY HIGH ENERGY $\gamma$-RAY OBSERVATIONS}

We start by making a brief summary of what has been claimed by other experiments observing at the highest energies.

The HEGRA IACT, using 4 years of data (from 1999 to 2002), found a source to the north of Cygnus X-3, steady in flux over the years, extended, with radius $6.2^{\prime} \pm 1.2_{\text {stat }}^{\prime} \pm$ $0.9_{\text {sys }}^{\prime}$, and exhibiting a hard energy spectrum with a photon index of $\Gamma=-1.9 \pm 0.1_{\text {stat }} \pm 0.3_{\text {sys }}$ (Aharonian et al. 2005). Its integral flux above $1 \mathrm{TeV}$ amounts to $\sim 5 \%$ of the Crab Nebula, assuming a Gaussian profile for the intrinsic source morphology. The center of the source position was determined quite accurately at $\alpha=20^{\mathrm{h}} 31^{\mathrm{m}} 57.0^{\mathrm{s}} \pm 6.2_{\text {stat }}^{\mathrm{s}} \pm 13.7_{\mathrm{sys}}^{\mathrm{s}}$ and $\delta=41^{\circ} 29^{\prime} 56.8^{\prime \prime} \pm 1.1_{\text {stat }}^{\prime} \pm 1.0_{\text {sys }}^{\prime}(\mathrm{J} 2000.0)$.

The Whipple collaboration reported an excess at the position of the HEGRA unidentified source $(3.3 \sigma)$ in their archival data of 1989 and 1990 (Lang et al. 2004), with a flux level of $12 \%$ of the Crab Nebula for $E>600 \mathrm{GeV}$. The detected flux is in conflict with the HEGRA flux level and steady nature of the source, assuming they all have the same origin. This large difference between the detected flux levels, if physical, might suggest episodic emission (with low duty cycle) or variability over timescale measured in years. Nevertheless, the existence of $\gamma$-ray variability is difficult to reconcile with the extended appearance of the source. The large difference might also be due in part to unspecified systematic errors on the flux determination. Recently, the Whipple collaboration reported new observations of this field done with their $10 \mathrm{~m}$ telescope for $65.5 \mathrm{hr}$ during 2003 and 2005 (Konopelko et al. 2007). Their data are consistent with either a pointlike or an extended source with less than $6^{\prime}$ angular size. Regarding the position, the HEGRA and the latest Whipple data are barely in agreement: their centers of gravity are $\sim 9^{\prime}$ apart, and only agree when adding up the spatial uncertainties in both data sets in opposite directions. Konopelko et al. do not provide a energy spectrum for this source, but give a $8 \%$ Crab-level flux (although with no energy threshold specified) under the assumption of a steep (Crab-like) energy spectrum.

The Cygnus region shows an excess in the MILAGRO data (Abdo et al. 2007). The flux at $20 \mathrm{TeV}$ in a $3^{\circ} \times 3^{\circ}$ region centered at the HEGRA position is $\left(9.8 \pm 2.9_{\text {stat }} \pm 2.7_{\text {sys }}\right) \times$ $10^{-15} \mathrm{TeV}^{-1} \mathrm{~cm}^{-2} \mathrm{~s}^{-1}$ assuming a differential energy spectrum $E^{-2.6}$. This flux is 3 times the HEGRA flux extrapolated at 20 $\mathrm{TeV}$. The Tibet air shower detector recently reported evidence for an excess also in their VHE $\gamma$-ray candidate set from this region (Amenomori et al. 2006).

In this rich observational and theoretical context we report here on MAGIC telescope observations of TeV J2032+4130.

\section{MAGIC OBSERVATIONS AND RESULTS}

The MAGIC single-dish Imaging Air Cerenkov Telescope (see, e.g., Cortina et al. 2005 for a detailed description) is located on the Canary Island of La Palma. Its angular (energy) resolution is approximately $0.09^{\circ}(20 \%)$, and the trigger (analysis) threshold is 55 (60) $\mathrm{GeV}$ at zenith in dark conditions (see Albert et al. 2008). One of the unique characteristics of MAGIC is its capability of observing under moderate moonlight illumination (Albert et al. 2007a), albeit with a slightly elevated threshold.

The field of view of $\mathrm{TeV} \mathrm{J} 2032+4130$ was observed with MAGIC for more than $100 \mathrm{hr}$ distributed in 2005, 2006, and 2007 (see Table 1). During the first period in summer 2005, the observation was carried out in ON/OFF mode, that is, the source was observed on-axis while observations from an empty, nearby field of view were used to estimate the background. In summer 2006 and 2007, the data were taken in Wobble mode, using five positions around the HEGRA position instead of the usual two symmetrical position in order to monitor a wider field of view. Quality cuts based on the trigger and after-cleaning rates were applied in order to remove bad weather runs and data spoiled by car or satellite light flashes. After these quality cuts the total observation time is $93.7 \mathrm{hr}$. The energy range for which we report these results is significantly above the aforementioned trigger and analysis threshold energies due to the fact that the observations were scheduled during moonlight and at relatively high zenith angles (up to $44^{\circ}$ ).

The data analysis was carried out using the standard MAGIC analysis and reconstruction software (Bretz \& Wagner 2003). It follows the general stream explained in Albert et al. (2006b, $2006 c, 2006 d)$. After calibration and two levels of image cleaning tail cuts (for image core and boundary pixels, see Fegan 1997), the camera images are parameterized by the so-called image parameters (Hillas 1985). The random forest method was applied for the $\gamma /$ hadron separation (Albert et al. 2007b). Using this method a parameter, dubbed hadronness $(\mathrm{H})$, can be calculated for every event and is a measure of the probability 


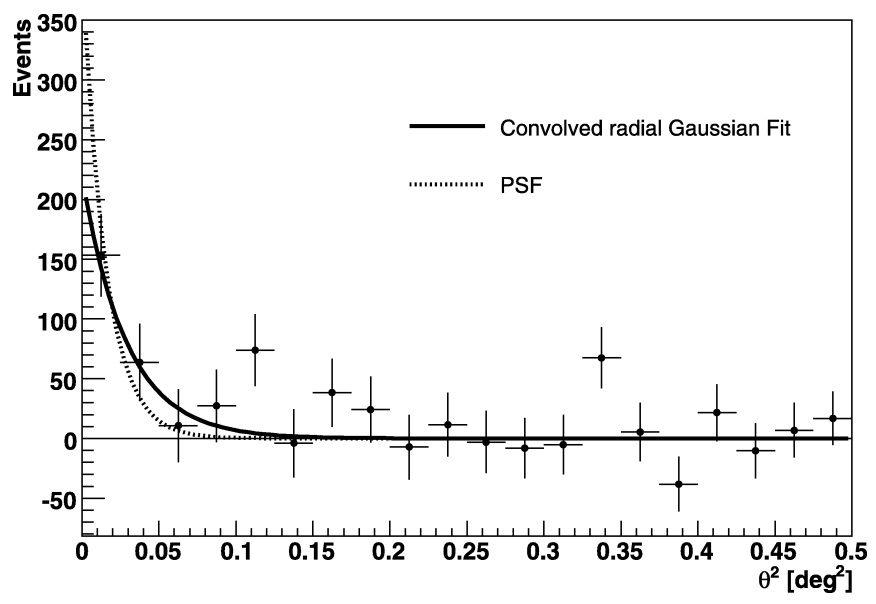

FIG. 1.-Distribution of the $\theta^{2}$-parameter for events coming from the direction of TeV J2032+4130 (SIZE > 800 photoelectrons), the background distribution subtracted (black points). A convolved radial Gaussian fit $F=$ $A \exp \left[-0.5 \theta^{2} /\left(\sigma_{\mathrm{psf}}^{2}+\sigma_{\mathrm{scc}}^{2}\right)\right]$ is indicated by the solid black line with $\sigma_{\mathrm{src}}=5.0^{\prime}$ $\pm 1.7^{\prime}$. The $\sigma_{\mathrm{psf}}$ was measured from MC simulation and validated with Crab Nebula observations to be $\sigma_{\mathrm{psf}}=5.2^{\prime} \pm 0.1^{\prime}$ (dashed black line).

that the event is not $\gamma$-like. The $\gamma$-like sample is selected for images with $\mathrm{H}$ below a specified value, which is optimized using a sample of Crab Nebula data processed with the same analysis stream. An independent sample of Monte Carlo $\gamma$ showers was used to determine the cut efficiency. Since part of our observations was recorded during partial moonshine, we have corrected the efficiency loss due to the increase of ambient light following the procedure outlined in Albert et al. (2007a).

The $\theta^{2}$-distribution was calculated, $\theta$ being the angular distance between the source direction and the reconstructed arrival direction of the showers. The reconstruction of individual $\gamma$ ray arrival directions makes use of the DISP method (DomingoSantamaria et al. 2005). The expected number of background events is calculated using five regions symmetrically placed for each wobble position with respect to the center of the camera and referred to as antisources. Figure 1 shows the distribution of the $\theta^{2}$ parameter for the excess observed from the direction of the source, for a SIZE cut of 800 photoelectrons. This relatively high SIZE cut was selected in order to optimize the sensitivity for a source with such a hard energy spectrum observed during moonlight. Therefore, the total number of $\gamma$ like excesses after Hillas cuts and applying a cut in $\theta^{2}<0.05$ is $N_{\text {ex }}=233$, for which a total significance of $5.6 \sigma$ is obtained. Table 2 shows the number of excesses above background for the different observing periods.

The excess is fitted to a Gaussian function folded with the telescope PSF, as obtained from Monte Carlo simulations and validated with Crab Nebula observations. The source is extended with respect to the MAGIC PSF. Its intrinsic size as-

TABLE 2

Events RECORDED ABOve SIZE $>800$ Photoelectrons

\begin{tabular}{crrrrr}
\hline \hline Year & $N_{\text {on }}$ & $N_{\text {off }}$ & $N_{\text {ex }}$ & $f_{\text {norm }}$ & $N_{\sigma}$ \\
\hline $2005 \ldots \ldots \ldots$ & 641 & 576 & 65 & 0.47 & 2.2 \\
$2006 \ldots \ldots \ldots$ & 688 & 559 & 129 & 0.20 & 4.8 \\
$2007 \ldots \ldots \ldots$ & 175 & 136 & 39 & 0.20 & 2.9 \\
Overall $\ldots \ldots$ & 1504 & 1271 & 233 & 0.27 & 5.6 \\
\hline
\end{tabular}

Notes. $-N_{\text {on }}, N_{\text {off }}$, and $N_{\text {ex }}$ refer to the number of events recorded in the direction of the source, the normalized background, and the $\gamma$-ray excess, respectively. The normalization ratio $f_{\text {norm }}$ and significance $N_{\sigma}$ are also shown.

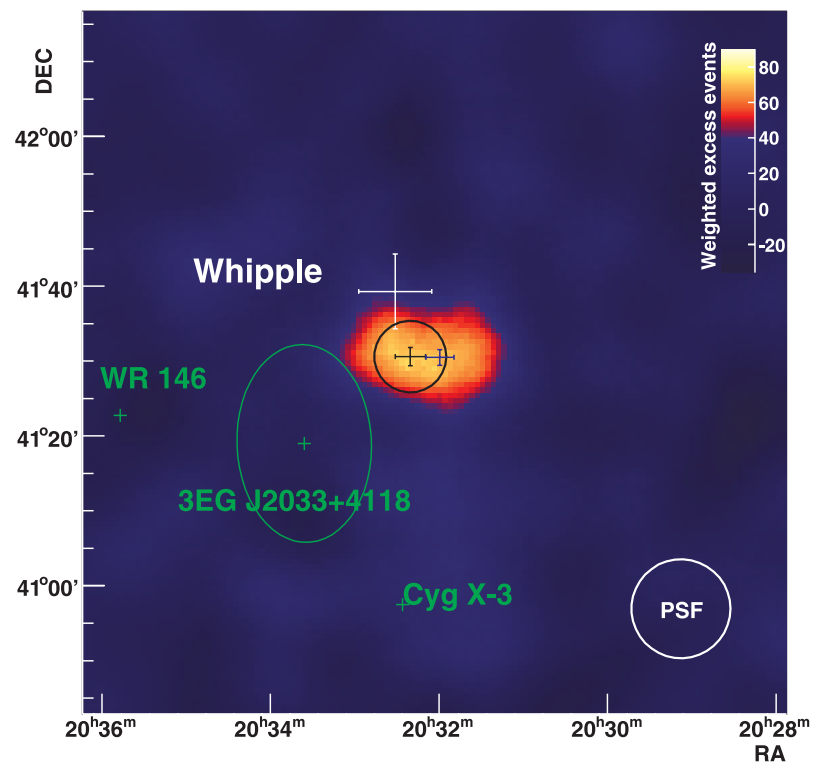

FIG. 2.-Gaussian-smoothed $\left(\sigma=4^{\prime}\right)$ map of $\gamma$-ray excess events (background subtracted) for energies above $500 \mathrm{GeV}$. The MAGIC position is shown with a black cross. The surrounding black circle corresponds to the measured $1 \sigma$ width. The last position reported by Whipple is marked with a white cross while the HEGRA position is shown with a blue cross in the center of the field of view. The error bars, in all cases, correspond to the linear sum of the statistical and systematic errors. The green crosses correspond to the positions of Cyg X-3, WR 146, and the EGRET source 3EG J2033+4118. The ellipse around the EGRET source marks the $95 \%$ confidence contour.

suming a Gaussian profile is $\sigma_{\text {src }}=5.0^{\prime} \pm 1.7_{\text {sta }}^{\prime} \pm 0.6_{\text {sys. }}^{\prime}$. The exact shape of the source, even if similar to the $\mathrm{keV}$ diffuse emission reported by Horns et al. (2007), cannot be completely trusted due to limited statistics and telescope pointing systematics.

Figure 2 shows the Gaussian-smoothed $\left(\sigma=4^{\prime}\right)$ map $\left(0.65^{\circ}\right.$ $\times 0.65^{\circ}$ ) of $\gamma$-ray emission (background subtracted) around $\mathrm{TeV} \mathrm{J} 2032+4130$ for energies $E>500 \mathrm{GeV}$. The position of a few previously observed $\gamma$-ray source candidates are also shown, namely, Cyg X-3, the EGRET source 3EG J2033+4118 (with its confidence contour at 95\%), the Wolf-Rayet star WR 146, and the Whipple and HEGRA experimental positions. The regions around Cyg X-3, WR 146, and 3EG J2033+4118 have been further investigated by us and no detection is obtained for a steady emission. The upper limit fluxes (Rolke et al. 2005), for a $95 \%$ confidence level, above $500 \mathrm{GeV}$ for a pointlike source at these positions are given in Table 3 .

To determine the best position of the MAGIC detection the excess map was fitted to a 2D bell-shaped function. The result is shown in the sky map with a black cross and a circle indicating its size. The best-fit coordinates are R.A. $=20^{\mathrm{h}} 32^{\mathrm{m}} 20^{\mathrm{s}}$ $\pm 11_{\text {stat }}^{\mathrm{s}} \pm 11_{\text {sys }}^{\mathrm{s}}$ and decl. $=41^{\circ} 30^{\prime} 36.0^{\prime \prime} \pm 1.2_{\text {stat }}^{\prime} \pm 1.8_{\text {sys }}^{\prime}$ (J2000.0; for more details on the systematic uncertainties in the source position determination, see Bretz \& Wagner 2003).

TABLE 3

UPPER LiMIT FOR SOURCES IN THE FOV, ABOVE $500 \mathrm{GeV}$

\begin{tabular}{cc}
\hline \hline Target Name & $\begin{array}{c}\text { Flux } \\
\text { (Crab Nebula) }\end{array}$ \\
\hline Cyg X-3 $\ldots \ldots \ldots \ldots \ldots \ldots$ & 0.011 \\
WR $146 \ldots \ldots \ldots \ldots \ldots$ & 0.010 \\
3EG J2033+4118 ...... & 0.009 \\
\hline
\end{tabular}


The position found is compatible within errors with the one determined by HEGRA, and barely compatible with the claims by Whipple mentioned above (in Konopelko et al. 2007).

The TeV J2032+4130 energy spectrum was obtained using the Tikhonov unfolding technique (Tikhonov \& Arsenin 1979). It can be fitted $\left(\chi^{2} /\right.$ dof $\left.=0.3\right)$ by a power-law function. The differential flux $\left(\mathrm{TeV}^{-1} \mathrm{~cm}^{-2} \mathrm{~s}^{-1}\right)$ is

$$
\frac{d N}{d E d A d t}=(4.5 \pm 0.3) \times 10^{-13}\left(\frac{E}{1 \mathrm{TeV}}\right)^{-2.0 \pm 0.3}
$$

The errors quoted are only statistical. The systematic error is estimated to be $35 \%$ in the flux level and 0.2 in the photon index (see Albert et al. 2008). The differential energy spectrum is shown in Figure 3. The HEGRA TeV J2032+4130 and MAGIC Crab Nebula measured spectra (in Albert et al. 2008) are shown with the blue solid line and black dotted line, respectively. The MAGIC energy spectrum is compatible both in flux level and photon index with the one measured by HEGRA.

Crab Nebula data from the same periods and zenith angle distributions were studied with the same analysis chain to check for any systematic deviation due to the long observation period. No indication of time variability was observed: the source integral flux is constant within errors, at $3 \%$ of the Crab Nebula flux. The relative systematic uncertainty in the ratio of both fluxes was estimated to be less than $10 \%$. This uncertainty comes mainly from the slightly different atmospheric transmission conditions and differences in the detector parameters during data taking of the source and the Crab Nebula.

For illustrative purposes, the dotted lines in Figure 3 represent one-zone hadronic and leptonic models of the high-energy emission, both consistent with observations at lower energies in the region. Under the hadronic scenario, the $\pi^{0}$ are obtained from a proton parent population described by a power law (index $\Gamma=-2$ ) with exponential cutoff at $100 \mathrm{TeV}$. The cutoff value was adopted to be consistent with the upper limit at the highest energies coming from the HEGRA spectrum. The inverse Compton spectrum is obtained from an electron population with equal index and a $40 \mathrm{TeV}$ exponential cutoff scattering off the CMB photons. As in Aharonian et al. (2005), we do not consider here the conditions under which particles are accelerated or how they lose energy. Our leptonic fits (see also the quoted paper for an SED representation) can only cope

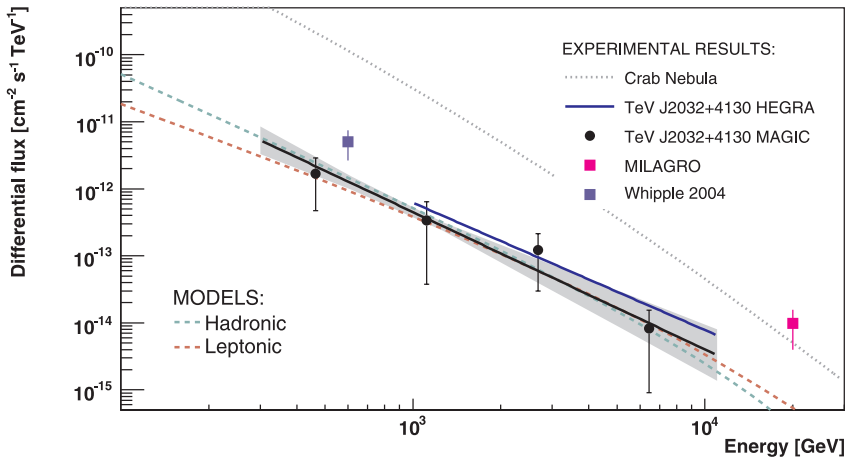

FIG. 3.-Differential energy spectrum from TeV J2032+4130 as measured by the MAGIC telescope in black solid line. The gray shadow shows the $1 \sigma$ error in the fitted energy spectrum. The flux observed by Whipple in 2005 and in the MILAGRO scan are marked with colored squares (blue and pink, respectively). The gray dotted line represents the Crab Nebula energy spectrum measured by MAGIC. The blue line shows the HEGRA energy spectrum. Theoretical one-zone model predictions are depicted with dashed lines.

with the data if we are actually looking at a Compton peak around the energy range of detection, which is not fully discarded within errors. Both models are compatible with the highenergy emission. Confirming the reality of the diffuse emission detected at lower energies is crucial to distinguish between these and more complex models.

\section{CONCLUDING REMARKS}

MAGIC observations confirm the location of $\mathrm{TeV}$ J2032+4130 found by HEGRA. The MAGIC observation shows an extended source with a significance of $5.6 \sigma$. We find a steady flux with no significant variability within the 3 year span of the observations (with the flux being at a similar level to the HEGRA data of the period 2002-2005). We also present the source energy spectrum obtained with the lowest energy threshold to date, which, within errors, is compatible with a single power law.

We thank the IAC for the excellent working conditions at the ORM. The support of the German BMBF and MPG, the Italian INFN, the Spanish CICYT, the ETH Research Grant TH 34/04 3, and the Polish MNiI Grant 1P03D01028 is gratefully acknowledged.

\section{REFERENCES}

Abdo, A. A., et al. 2007, ApJ, 658, L33

Aharonian, F. A., et al. 2002, A\&A, 393, L37

2005, A\&A, 431, 197

2006, A\&A, 460, 743

Albert, J., et al. 2006a, Science, 312, 1771

2006b, ApJ, 637, L41

2006c, ApJ, 638, L101

2006d, ApJ, 643, L53

2007a, Astropart. Phys., submitted (astro-ph/0702475)

2007b, Astropart. Phys., submitted (arXiv:0709.3719)

2008, ApJ, in press (arXiv:0705.3244)

Amenomori, M., et al. 2006, Science, 314, 439

Anchordoqui, L. A., et al. 2007, Phys. Rev. D, 75, 063001

Bednarek, W. 2003, MNRAS, 345, 847

Bosch-Ramon, V., Aharonian, F., \& Paredes, J. M. 2005, A\&A, 432, 609

Bretz, T., \& Wagner, W. 2003, in Proc. 28th Int. Cosmic Ray Conf. (Tsukuba), 2947

Butt, Y., et al. 2003, ApJ, 597, 494

2006, ApJ, 643, 238
Cortina, J., et al. 2005, in Proc. 29th Int. Cosmic Ray Conf. (Pune), 5, 359

Domingo-Santamaría, E., \& Torres, D. F. 2006, A\&A, 448, 613

Domingo-Santamaría, E., et al. 2005, in Proc. 29th Int. Cosmic Ray Conf. (Pune), 5, 363

Fegan, D. J. 1997, J. Phys. G, 23, 1013

Hillas, A. M. 1985, in Proc. 19th Int. Cosmic Ray Conf. (La Jolla), 3, 445

Horns, D., et al. 2007, A\&A, 469, L17

Konopelko, A., et al. 2007, ApJ, 658, 1062

Lang, M. J., et al. 2004, A\&A, 423, 415

Martí, J., et al. 2006, A\&A, 451, 1037 2007, A\&A, 472, 557

Mukherjee, R., et al. 2003, ApJ, 589, 487

Paredes, J. M., et al. 2007, ApJ, 654, L135

Rolke, W. A., López, A. M., \& Conrad, J. 2005, Nucl. Instrum. Methods Phys. Res., A551, 493

Tikhonov, A. N., \& Arsenin, V. Ja. 1979, in Numerical Methods for the Solution of Ill-posed Problems (Moscow: Nauka)

Torres, D. F., Domingo-Santamaría E., \& Romero, G. E. 2004, ApJ, 601, L75 